\title{
On mixing angles and magnetic moment of heavy tau neutrino
}

\author{
S.N.Gninenko円 \\ Institute for Nuclear Research, Russian Academy of Sciences, \\ Moscow 117 312, Russia
}

\begin{abstract}
If the magnetic moment of unstable tau neutrinos with the mass of $O(\mathrm{MeV})$ is in the region of $10^{-8} \mu_{B} \lesssim \mu_{\nu_{\tau}} \lesssim 10^{-6} \mu_{B}$, it is compatible with the present experimental and cosmological bounds.

It is pointed out here, that if the tau neutrino has such a large magnetic moment and can oscillate into a neutrino of another flavour the results from $\nu e$ scattering experiment at LAMPF constrain the tau neutrino mixing angles to $\sin ^{2} 2 \theta_{e \tau} \lesssim 2 \times 10^{-6}-2 \times 10^{-2}$ and $\sin ^{2} 2 \theta_{\mu \tau} \lesssim 10^{-6}-10^{-2}$ depending on the magnetic moment value in the allowed region.
\end{abstract}

In many extensions of the standard model the neutrino acquire a nonzero mass and a magnetic moment (for review see e.g. [1]). Usually large magnetic moment imply large masses. In the simplest extension, for example, neutrino masses and magnetic moment are proportional [2, 3]. A nonzero mass of the neutrino is also required for Cosmology to solve the problem of dark matter in the Universe. It is generally assumed that massive tau neutrinos are natural candidates for the hot component of the dark matter of the Universe, see e.g. ref. [4]. But they cannot provide an explanation for a cold dark matter, because of constraints from relic abundance. However, as was proposed by Giudice [5], a stable (or quasi-stable in cosmological times) tau neutrino with a mass in the range from $\simeq 1 \mathrm{MeV}$ up to the present experimental upper limit of $25 \mathrm{MeV}$ and a magnetic moment $\mu_{\nu_{\tau}}$ as large as $\simeq 10^{-6}$ Bohr magneton $\left(\mu_{B}=e / 2 m_{e}\right)$ could be a possible candidate for a cold dark matter particle.

Experimental limit from BEBC (CERN), $\mu_{\nu_{\tau}}<5.4 \times 10^{-7} \mu_{B}$ [6], and revised calculations of the evolution and freeze-out of the tau neutrino number density, ref. [7], definitely rule out Giudice's hypothesis. However, it was pointed out, that if the tau neutrino is an unstable particle with the lifetime exceeding one second, a band of magnetic moment values

$$
10^{-8} \mu_{B} \lesssim \mu_{\nu_{\tau}} \lesssim 10^{-6} \mu_{B}
$$

remains compatible with experimental and cosmological bounds [7].

In this case, if the magnetic moment of $\nu_{\tau}$ exists, massive tau neutrinos could manifest themselves in terrestrial experiments through the effect of $\nu_{\mu}\left(\nu_{e}\right) \rightarrow \nu_{\tau}$

\footnotetext{
${ }^{1}$ E-mail address: Sergei.Gninenko@cern.ch
} 
neutrino oscillations [8], or, since magnetic moment value of $10^{-8} \mu_{B} \lesssim \mu_{\nu_{\tau}} \lesssim$ $10^{-6} \mu_{B}$ is large enough, through tau neutrino electromagnetic interactions, or through combined effect.

In the recent paper, ref. [9], it was shown that combined existence of $\nu_{\mu} \rightarrow$ $\nu_{\tau}$ and/or $\nu_{e} \rightarrow \nu_{\tau}$ oscillations and a large nonzero magnetic moment of the tau neutrino would increase the total rate of events in $\nu_{\mu}\left(\nu_{e}\right)$ neutrino- electron scattering experiments. The basic idea is rather simple.

Assuming that a muon neutrino beam has a component of tau neutrinos due to $\nu_{\mu} \rightarrow \nu_{\tau}$ oscillations. In case of two-neutrino mixing neutrino states evolve with a time $t$ as

$$
|\nu>(t)=a(t)| \nu_{\mu}>+b(t) \mid \nu_{\tau}, \mu_{\nu_{\tau}} \neq 0>
$$

where $\left|\nu_{\mu}\right\rangle$ and $\left|\nu_{\tau}\right\rangle$ denote weak eigenstates of $\nu_{\mu}$ and $\nu_{\tau}$ neutrinos, and $a^{2}(t), b^{2}(t)$ are the probabilities to find $\nu_{\mu}$ or $\nu_{\tau}$ in the beam at a given moment $t$, respectively. It is assumed that $a^{2}(0)=1$ at $t=0$. The probability $b^{2}(t)$ depends on the parameters of $\nu_{\mu}-\nu_{\tau}$ oscillations as [10]:

$$
b^{2}\left(t \simeq \frac{L}{c}\right)=P\left(\nu_{\mu} \rightarrow \nu_{\tau}\right)=\sin ^{2} 2 \theta_{\mu \tau} \sin ^{2} \frac{\Delta m^{2} L}{4 E}
$$

or

$$
P\left(\nu_{\mu} \rightarrow \nu_{\tau}\right) \approx \sin ^{2} 2 \theta_{\mu \tau} \sin ^{2} \frac{1.27 \Delta m^{2}\left(e V^{2}\right) L(k m)}{E(G e V)}
$$

where $\sin ^{2} 2 \theta_{\mu \tau}$ is the mixing angle, and $\Delta m^{2}=\left|m_{3}^{2}-m_{2}^{2}\right|$ is the difference of squares of the mass eigenstates in $e V^{2}, E$ is the neutrino energy in $\mathrm{GeV}$, and $L$ is the mean distance between the neutrino source and the detector in $\mathrm{km}$. In the above formulas it is also assumed that magnetic field $B$ is weak enough not to affect the probability of oscillations $P\left(\nu_{\mu} \rightarrow \nu_{\tau}\right)$, i.e. $\Delta m^{2} / 2 E \gg \mu_{\nu_{\tau}} B$ [11].

Then if magnetic moment of the $\nu_{\tau}$ exists, it will contribute to a non-coherent part of the $\nu_{\tau} e^{-}$scattering cross section via the reaction that change the helicity of the tau neutrino (hence right-handed neutrino states should exist). This might result in observable deviations from purely $\nu_{\mu} e^{-}$electro-weak reaction which is well predicted by the SM. Indeed, since the electromagnetic cross section is orders of magnitude larger than the weak cross section, even a small fraction of tau neutrinos with nonzero magnetic moment in the muon neutrino beam could lead to an observable effects in $\nu_{\mu} e^{-}$scattering, while the magnetic moment of muon neutrino could be small enough to contribute effectively to $\nu_{\mu} e^{-}$scattering. The production rate of isolated electrons via $\nu_{\tau} e^{-}$scattering in the detector 
depends also on the probability $P\left(\nu_{\mu} \rightarrow \nu_{\tau}\right)$ to find a $\nu_{\tau}$ neutrino in the neutrino beam. This probability can be calculated from the neutrino survival and transition probabilities using Eqs. $(3,4)$.

This effect can be used to constrain mixing angles of the tau neutrino with neutrino of another flavours. It was shown, ref. [9], that using the results obtained from the experiment on study of the $\nu_{e} e^{-}$elastic scattering at LAMPF, ref. [12], the following combined limits on the mixing angles and tau neutrino magnetic moment for $\Delta m^{2} \gtrsim 10 \mathrm{eV}^{2}$ can be derived

$$
\begin{aligned}
& \sin ^{2} 2 \theta_{e \tau} \times \mu_{\nu_{\tau}}^{2} \leq 2.3 \times 10^{-18} \mu_{B}^{2} \\
& \sin ^{2} 2 \theta_{\mu \tau} \times \mu_{\nu_{\tau}}^{2} \leq 1.1 \times 10^{-18} \mu_{B}^{2}
\end{aligned}
$$

Note, that if the tau neutrino mass is in the range from $\simeq 1$ to $25 \mathrm{MeV}$ then

$$
\Delta m^{2}=\left|m_{3}^{2}-m_{2}^{2}\right| \gg 10 e V^{2}
$$

since the $m_{2}$ mass is experimentally measured to be smaller than $160 \mathrm{KeV} / c^{2}$ [13.

Assuming the tau-neutrino magnetic moment to be in the range of $10^{-8} \mu_{B} \lesssim$ $\mu_{\nu_{\tau}} \lesssim 10^{-6} \mu_{B}$ the following limits on the mixing angles can obtained from Eqs. $(5,6)$

$$
\begin{aligned}
& \sin ^{2} 2 \theta_{e \tau} \lesssim 2 \times 10^{-6} \quad \mu_{\nu_{\tau}} \sim 10^{-6} \mu_{B} \\
& \sin ^{2} 2 \theta_{e \tau} \lesssim 2 \times 10^{-2} \quad \mu_{\nu_{\tau}} \sim 10^{-8} \mu_{B} \\
& \sin ^{2} 2 \theta_{\mu \tau} \lesssim 10^{-6} \quad \mu_{\nu_{\tau}} \sim 10^{-6} \mu_{B} \\
& \sin ^{2} 2 \theta_{\mu \tau} \lesssim 10^{-2} \quad \mu_{\nu_{\tau}} \sim 10^{-8} \mu_{B}
\end{aligned}
$$

The limits are valid for the mass region $\Delta \mathrm{m}^{2} \gtrsim 10 \mathrm{eV}^{2}$ which is consistent with the mass region given by Eq.(7). They are obtained under assumption of combined existence of neutrino oscillations and a (large) magnetic moment of the tau neutrino. Nevertheless, it is interesting to compare them with the present experimental limits of $\sin ^{2} 2 \theta_{e \tau}<0.15$ obtained from $\overline{\nu_{e}} \rightarrow \nu_{x}$ disappearance reactor experiments in Bugey [14] and in Krasnoyarsk [15], or $\sin ^{2} 2 \theta_{\mu \tau}<0.01$ for $\nu_{\mu} \rightarrow \nu_{\tau}$, obtained from the exclusion plot of ref. 16] for $\Delta m^{2}>10 \mathrm{eV}$. For $\mu_{\nu_{\tau}} \sim 10^{-6} \mu_{B}$, the limit from Eq.(10) is also more stringent than the preliminary experimental limits of $\sin ^{2} 2 \theta_{\mu \tau}<3.5 \times 10^{-3}$ from the CHORUS [17], or $\sin ^{2} 2 \theta_{\mu \tau}<3.7 \times 10^{-3}$ from the NOMAD [18] experiments at CERN obtained for the mass region $\Delta m^{2}>1000 e V^{2}$. 
On the other hand, if CHORUS or NOMAD will find $\nu_{\mu} \rightarrow \nu_{\tau}$ oscillations one would expect $\sin ^{2} 2 \theta_{\mu \tau} \simeq 10^{-4}$ for the large $\Delta m^{2}$. In this case, using Eqs. $(1,6)$ one can obtain that a small window around $\mu_{\nu_{\tau}} \sim O\left(10^{-7}\right) \mu_{B}$ might still be of interest for searching for tau neutrino magnetic moment.

\section{Acknowledgements}

I thank N.V. Krasnikov for useful discussions and G.Giudice for useful comment. 


\section{References}

[1] R.N. Mohapatra and P.B. Pal, in "Massive Neutrinos in Physics and Astrophysics", World Scientific, Singapore, 1991.

[2] B.W. Lee and R.E. Shrock, Phys. Rev. D16 (1977) 1444.

[3] W. Marciano and A.I. Sanda, Phys. Lett. B67 (1977) 303.

[4] S. Sarkar, Preprint hep-ph/9602260.

[5] G.F. Giudice, Phys. Lett. B251 (1990) 460;

G.F. Giudice, Mod. Phys. Lett. A6 (1991) 851.

[6] A.M. Cooper - Sarkar et al., Phys. Lett. B280 (1992) 153.

[7] D. Grasso and E.W. Kolb, Phys. Rev. D54 (1996) 1374;

L.H. Kawano et al., Phys. Lett. B275 (1992) 487.

[8] B. Pontecorvo, Zh. Eksp. Teor. Fiz. 33 (1957) 549; [JETP 6(1958)429].

[9] S.N. Gninenko, "Limits on $\nu_{\mu}\left(\nu_{e}\right) \rightarrow \nu_{\tau}$ oscillations and tau neutrino magnetic moment from neutrino-electron scattering", Preprint hep-ph/9712220, to appear in Phys. Lett. B.

[10] See e.g. review: S.M. Bilenky and B. Pontecorvo, Phys. Rep.41 (1978) 225.

[11] J.D. Vergados, Nucl. Phys. Proc. Suppl. 22A(1991) 21.

[12] D.A. Krakauer et al., Phys. Lett. B252 (1990) 177;

R.C. Allen et al., Phys. Rev. D47 (1993) 11.

[13] K. Assamagau et al., Phys. Lett. B335 (1994) 231.

[14] B. Achkar et al., Nucl. Phys. 434 (1995) 503.

[15] G.S. Vidyakin et al., JETP Lett. 59 (1994) 390.

[16] N. Ushida et al., Phys. Rev. Lett. 57 (1986) 2897.

[17] CHORUS collaboration, CERN-PPE/97-149, submitted to Phys. Lett. B (1998);

[18] A. Rubbia, "Neutrino Physics at Accelerators", Talk given at Lepton-Photon Symposium, Hamburg, 1997. 